\title{
The Influence of Part Dimensions and Tolerance Size to Trigger Characteristics
}

\author{
Martin Macko ${ }^{1,{ }^{*}}$ - Slobodan Ilić ${ }^{2}$ - Mirko Jezdimirović ${ }^{2}$ \\ 1 University of Defence, Czech Republic \\ 2 Defence University, Military Academy, Serbia
}

This article describes a negative effect of size tolerance on the trigger characteristic. The trigger characteristic is the dependence of trigger force and trigger angle. An invalid trigger characteristic affects the accuracy of shooting and can be changed by not only proper choice of dimensions, but also the location of individual components. The paper shows an example of trigger mechanism that is designed as a Glock type mechanism. The solution for designers of small arms authors suggested the use of software MW.

Keywords: dimensions, tolerance, accuracy, trigger mechanism, trigger characteristic

\section{O INTRODUCTION}

The problem of production accuracy and precision, as well as the shape and position of movable and immovable integral parts of assemblies and mechanisms is the topic of many papers. There are different approaches to this problem. On the one hand, a number of authors focus on the influential parameters, production of parts surfaces, while, on the other hand, some authors deal with the measurement of the load assemblies and components, and their influence on the overall product design. For example, in [1], the authors focus their attention on the changing axis of the tool position in relation to the surface to be processed by milling. Their target was achieving an increase in milling efficiency (improvement of functional surface properties, increase in milling accuracy, increase in tool durability, decrease in energy load on a machine, and shortening of milling time). Proper selection of monitoring points is of paramount importance for drawing conclusions about the design of products based on the measured parameters, regardless of the size scale. In paper [2], the authors had chosen the strain as the relevant influential parameter to measure. The aim of their study was to determine the forces acting on a foldable bicycle riding during various situations. For this purpose, the test bicycle was equipped with strain gauges connected in full Wheatstone bridges at eight measuring points.

Similar problems are met at the hand arms design. One of the main characteristics of hand arms is certainly the accuracy. Accuracy of shooting depends on various factors: the position of barrel muzzle during firing, shapes and types of bullets, atmospheric conditions, but especially, trigger mechanism displacements and forces balancing.
The first factor, the position of a muzzle, is directly affected by both, subjective, as well as objective parameters caused by mechanism design. The subjective parameters are related to the individual user - shooter. The proper aiming and possession of arms, especially the motion of the forefinger on the trigger during triggering (Fig. 1) is the most important.
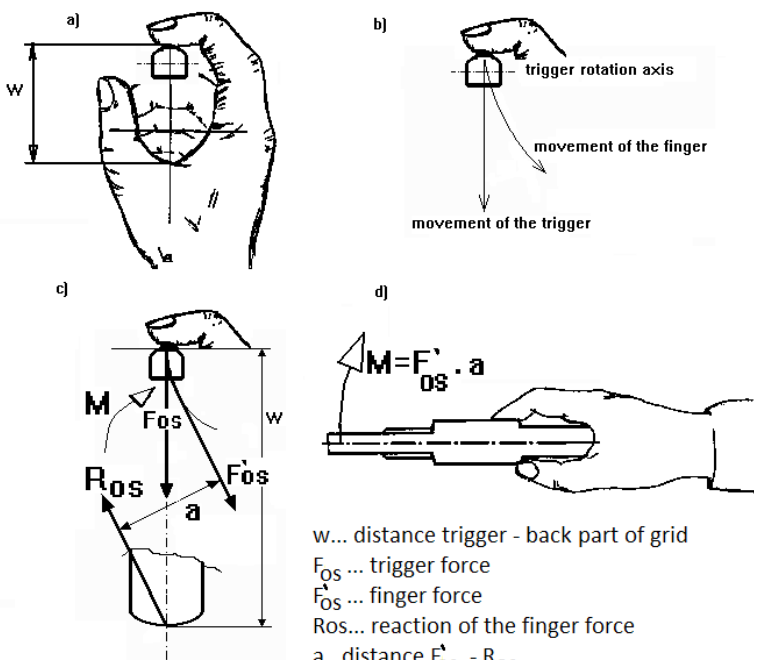

W... distance trigger - back part of grid

$\mathrm{F}_{\mathrm{OS}}$... trigger force

$\mathrm{F}_{\mathrm{OS}}^{\prime}$.... finger force

Ros... reaction of the finger force

a...distance $F_{O S}^{\prime}-R_{O S}$

M...moment of finger force $\mathrm{F}_{\mathrm{OS}}^{\circ}$

Fig. 1. The influence of forefinger motion on the trigger mechanism; a) usual forefinger position on the trigger, b) forefinger movement and trigger movement are different, c) different

forefinger movement and trigger movement causes moment of force $M, d$ ) Moment of force $M$ causes muzzle movement

The relation between the trigger mechanism and the shooter is content in fact that forefinger force acts on the trigger, during the process of triggering, changing the position of the barrel muzzle according to the triggering mechanisms internal forces and momentums [3] and [4]. Trigger force is equal to the resistance of the trigger. The value of force triggering 
is usually stated in the specifications of arms only as a (maximum) trigger force. Trigger force causes the movement of the barrel muzzle and the shooter with an adequate reaction, tries to keep the barrel position directed towards the line of sight.

Objective parameters related to the construction of arms parts and mechanisms, their position, dimensions and accuracy of production. The position of the barrel muzzle during the shooting process is under a direct influence of the trigger mechanism characteristics. The trigger characteristic represents dependence of trigger force on the trigger rotation angle. In each moment of the process, the firing trigger force has different values of $F_{o s}$ and direction of $F_{o s}$. One of the main designer task is to achieve optimal growth of the trigger characteristic and the correct choice of dimensions, positions and tolerances of trigger mechanism parts.

\section{STATE OF THE PROBLEM}

Arms designer should aim to achieve a smooth trigger characteristic, with slight force increasing, without a large rebounding of barrel. Such a change of force has an effect at least on the accuracy of shooting. Fig. 2 provides an overview of trigger force change and characteristic values of Fos for double action (DA) trigger mechanism, tested in paper [5]. These characteristics are given as an example of values for maximal trigger forces vs. trigger angles.

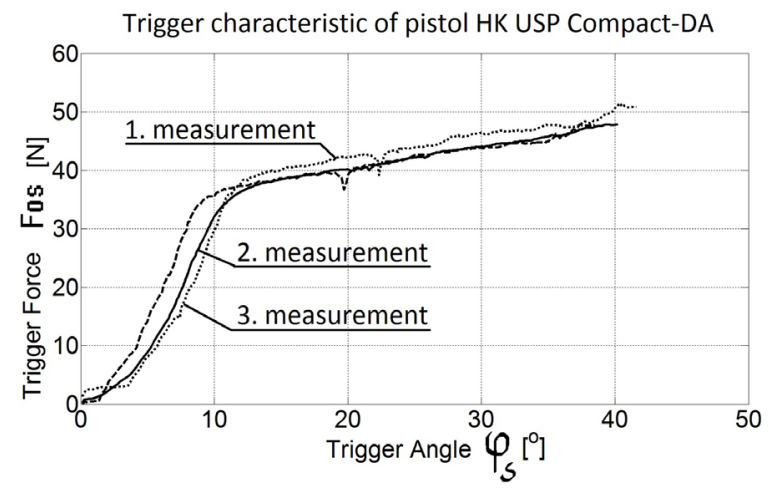

Fig. 2. Experimental trigger characteristics

The purpose of trigger mechanism is cocking and the release of impulse mechanism in a desired point of arms initial cup. The release of the impulse mechanism affects the movement of arms because the shooter affects the trigger by the force of the forefinger and hand holding of the arms is not stationary. Through the optimal trigger characteristic with help of smallest internal displacements, the designer should develop a right form of the trigger mechanism [6].

As an example of an analysis DA trigger mechanism similar mechanism of pistol Glock 17 (Fig. 3) with appropriate dimensions of mechanism parts [7] is taken.

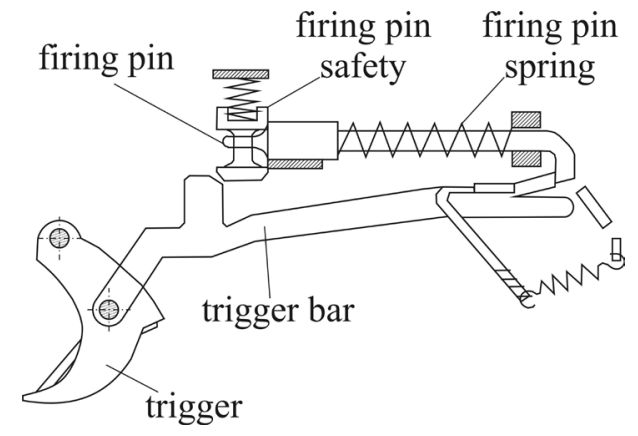

Fig. 3. Simulated firing mechanism

The trigger characteristic harvesting depends on many parameters. Fig. 4 shows the selected influential parameters (marked in capital letters) on the simulation model of trigger mechanism. Based on Fig. 4 the designer obviously has a vision about points and position to released function, loadings and initial displacements in the process of the triggering. Also, the main influence parameters are selected as the contact points in the mechanism during triggering which connects the internal mechanism design.

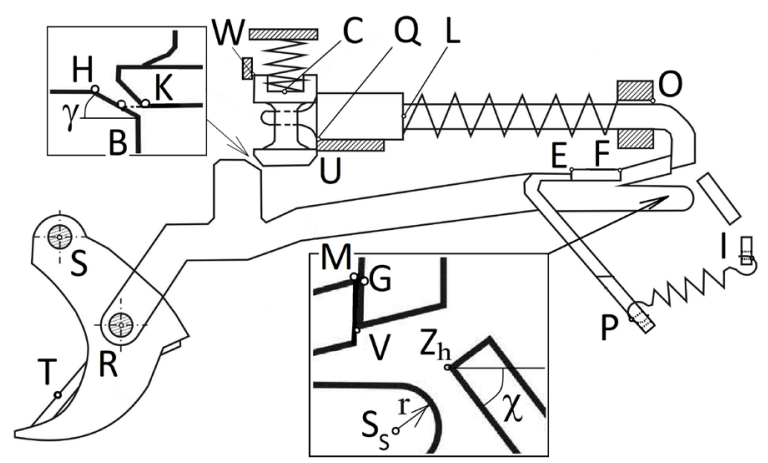

Fig. 4. Influential parameters on the trigger characteristic

This provides the designer with the capability to optimize dimensions and parts positions based on the criteria of trigger force maximum and/or trigger angle maximum. The solution of a simulation model provides a schematic view of the most important forces and the distances (Fig. 5) in the mechanism between parts of the mechanismand the direction of the parts displacements: $F_{o s}$ - trigger force, $F_{b}-$ reaction between trigger bar and firing pin safety, $F_{2}$ - firing pin safety force, $F_{3}$ - firing pin force, $F_{4}$ 
trigger bar spring force, $F_{m}$ - reaction between trigger bar and firing pin, $F_{z}$ - reaction between trigger bar and the inclined plane, $l$ - trigger bar horizontal displacement, $s_{m}$ - trigger bar vertical displacement equals $s_{z}, s_{b p}$ - firing pin displacement, $s_{c}$ - firing pin safety displacement, $\varphi_{s}$ - trigger angle, $\gamma$ - trigger bar inclinated plane angle, $\chi$ - inclinated plane angle.

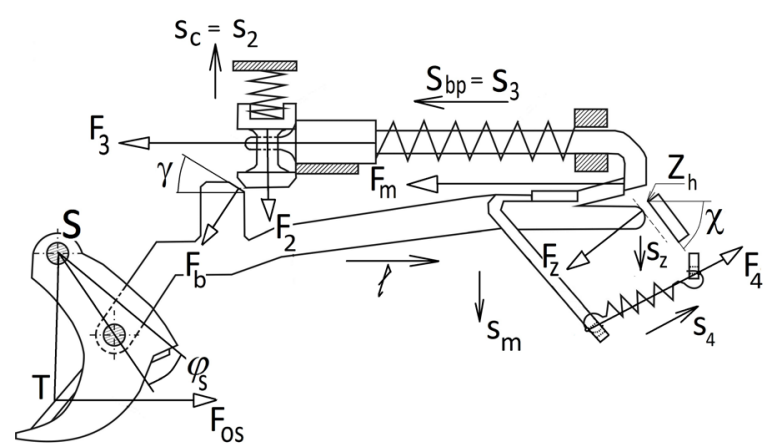

Fig. 5. Main forces, angles and displacement of the simulated mechanism

\section{SIMULATION TOOL}

There is various software for tolerance analysis or synthesis [7]:

Sigmund is a $3 \mathrm{D}$ tolerance analysis software package developed by Varatech and is fully integrated with major packages such as ProEngineer, SolidWorks, and SolidEdge.

MITCalc is designed in Microsoft Excel as an open system. MITCalc contains the design calculation tool: Tolerance analysis of various dimensional chains. The program solves the problem of tolerance analysis and optimization of a dimensional chain using the Worst case, the statistical RSS method, 6 Sigma method and the Monte Carlo.

TASysWorks is a 3D tolerance software package integrated with SolidWorks. It consists of two modules: TASysWorks for tolerance analysis and TASysWorksINTOL for tolerance synthesis in mechanical assemblies. TASysWorks allows four kinds of tolerance analysis: worst-case, RSS, Monte Carlo Simulation, Quadrature Technique and can provide linear and non-linear statistical analysis.

CETOL 6 Sigma is a software package integrated with both Pro/ENGINEER and CATIA V5. It allows users to model-analyze and allocate tolerances based on product performance requirements, while considering manufacturing process capabilities. It can handle three analysis models: derivative-based (Worst-case analysis, RSS analysis) and Monte Carlo simulation.
OptQuest is an optimization software tool that performs uncertainty and tolerance analysis through the process of defining constraints, specifying the objective of the outcome, and setting the requirements. OptQuest finds the optimal solution through Monte Carlo simulation. For tolerance synthesis the OptQuest works with a statistical distribution of component dimensions (the distribution gallery of OptQuest contains 12 different distributions) and the user enters the cost of components into the spreadsheet.

VarTran is a tolerancing package for tolerance analysis and optimization. It can perform all the standard analysis including statistical tolerancing, worst-case tolerancing, and sensitivity analysis. To optimize product or process performance VarTran provides three methods: maximize $C_{p k}$ (Process Capability Index), minimize the defective percent and Taguchi loss method.

For the analysis of dimensions, position, tolerance, forces and momentums as well as delays of the trigger mechanism, simulation components are tested by software solution MW [7]. The interface structure of the software package MW is shown in Fig. 6.

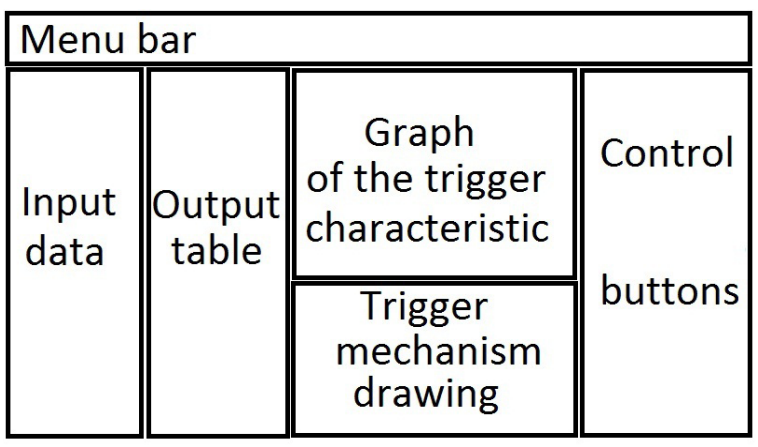

Fig. 6. The interface structure of software package MW for the simulation of triggering

\section{THE RESULTS OF THE SIMULATION}

The software solution of a simulation model gives designers the ability to analyze the forces, moments and displacement changes of individual points of the trigger mechanism in the design phase. This ensures an optimal distribution of trigger mechanism parts and a precise definition of the dimensions

In the aim to prove software model design which supported the date in Fig. 5 the initial input trigger force vs. angle of triggering is presented similarly to the experimental values in the Fig. 2. This was simulated for a similar mechanism of hand arm Glock 17. The diagram, (Fig. 7), shows the bouncing force 
peak of triggering force vs. the triggering angle on the mechanism, at the singular value of trigger angle, which is about 18 degrees. This form of characteristic is not welcome because during firing it has a negative influence on the jump of the barrel muzzle. The software simulation done in this paper to solve the causes in the mechanism of triggering determine this force peak as the main problem, which generates rebounding of barrel and arm.

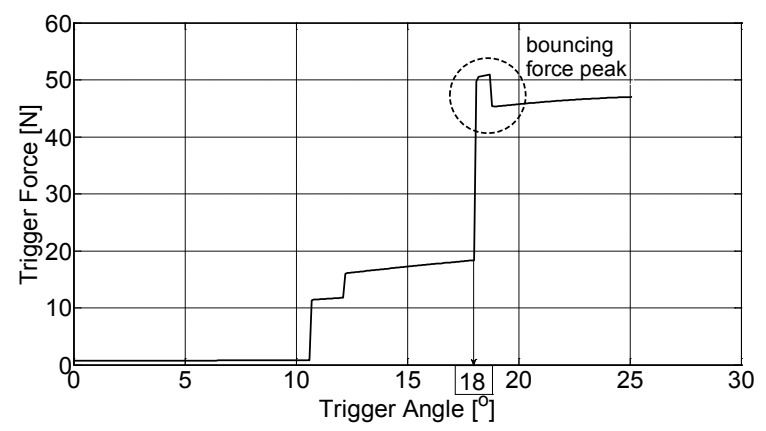

Fig. 7. The trigger characteristic for initial values

The basic concept of simulation provides displacement testing and forces vs. dimensions, shown in Fig. 5, and estimations which one is decisionally important for proper functioning and operation. Obvious disturbance is shown in the Fig. 7 absent in Fig. 2 correlated to the input or output force vs. input triggering angle. The input force is the trigger force $F_{O S}$. The output force of the mechanism is firing pin force $F_{3}$ (Fig. 5). The relation between these forces vs. trigger angle is shown in Fig. 8. For the fixed values of mechanism parts dimensions, these two forces relation vs. angle are not changed. Also, disturbing jump of trigger force remains on $F_{3}$ curve. This orientated that the main influence on the trigger force lays in dimensions and tolerances of input and output coordinates.

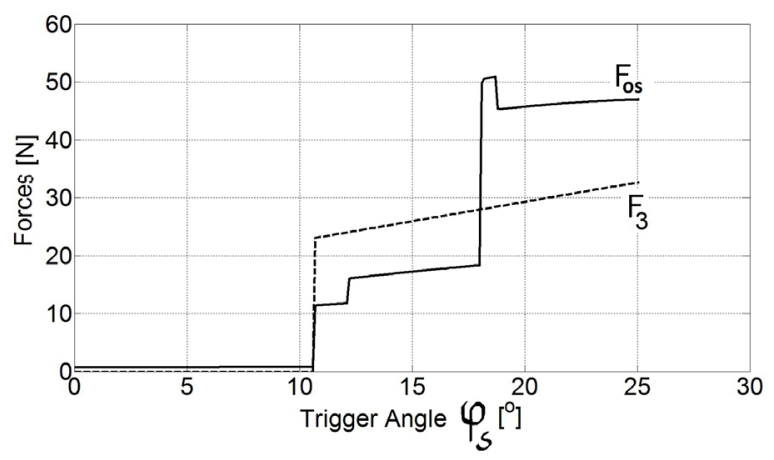

Fig. 8. Relation of input and output forces vs. trigger angle $\left(F_{3}\right.$ dashed curve, $F_{0 S}$ solid curve)
Fig. 9 represents basic displacement coordinates of mechanism parts which determine main force $F_{3}$ of arms operation function. Delay trigger angle $\Delta \varphi$ orientated instant of releasing firing pin and tolerances field which determines this as threshold for arms rebounding caused by jump of trigger force.

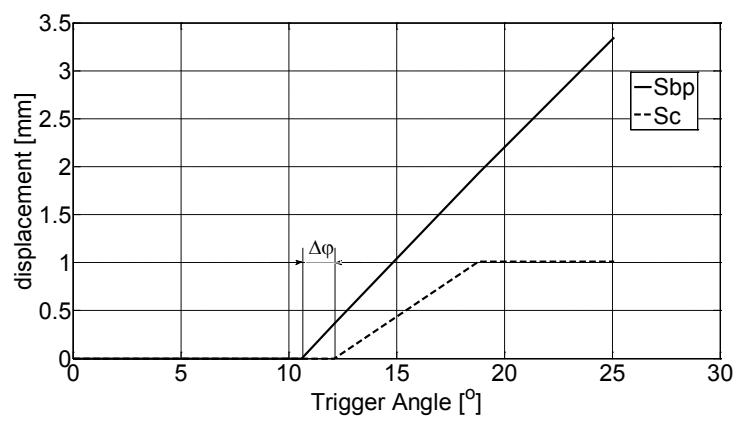

Fig. 9. Changes of displacement vs. trigger angle

A position of the $K$-point (Fig. 4) is very important for this type of the trigger mechanism. Change of $x$-position $K$ (coordinate system is determined by help of rotation point of the trigger $S$ : $x$-position point $S$ is $100 \mathrm{~mm}$ and $y$-position point $S$ is $100 \mathrm{~mm}$ ) allows to provide so called "timing" of firing pin releasing. It means in tolerance field causes the changes of firing pin shift instant and determining of safety, out of function operation affecting. In the Fig. 8 is showed a shape of trigger characteristic (solid curve) for initial dimensions (according to technical drawing) of the simulation while in the Fig. 10 are showed two examples - results of the simulation: the solid curve is for shooter acceptable shape of the trigger characteristic and dashed curve shows wrong shape. The coordinate of $x$-position $K$ was varied from 117.82 to $117.40 \mathrm{~mm}$. The first result of the simulation is showed in the Fig. 10 for the $x$-value 117.66 of the $K$ point: solid curve of trigger force does not a jump in the trigger angle of 18 degrees. This is acceptable solution for shooting.

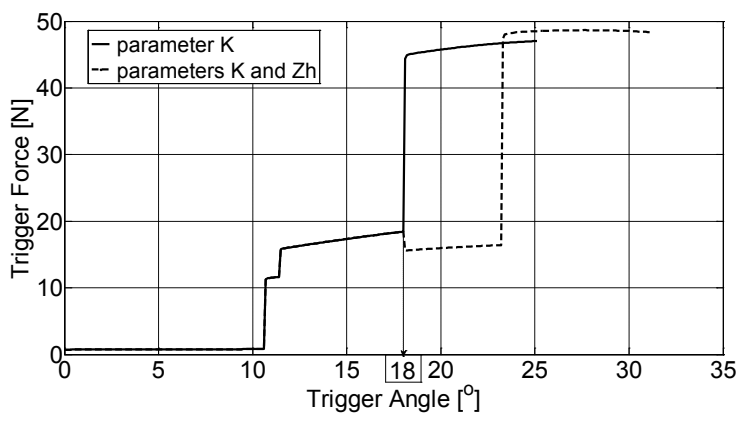

Fig. 10. Change of triggering force - variation of the parameters $K$ and $Z_{h}$ 
The second example of the simulation in the Fig. 10 (dashed curve) shows the change of the $y$-position $Z_{h}$ (x-position of the $K$-point was kept $117.66 \mathrm{~mm}$ from the first example). For change of the $y$ - position $Z_{h}$ from 117.53 to $118.53 \mathrm{~mm}$ the impact on the trigger characteristic is too big: the change causes the shift of the firing pin release time (delaying).

It is possible to choose more variations of different parameters in order to find the right shape of the trigger characteristic.

\section{CONCLUSION}

A designer should provide a tolerance analysis or tolerance synthesis in the frame of the preliminary design phase of the mechanisms design [8]. It is possible to solve this problem automatically by various software or by analytical considerations for the main string parameters which determinate the goal function. By using different software packages a designer can achieve a way of quicker and simpler provision of optimal solutions for assemblies and its size tolerances related to the function. Using results of the analytical study achieves a more effective design of the trigger mechanism by reason of observing the condition for acceptable trigger characteristics. Using special software for the simulation of the triggers or other mechanisms it is possible to gain information about all the parameters that influence trigger function. This means not only size, tolerances, positions but also forces, coefficients of friction, etc. This way takes into account, the so called instant delay of the mechanism, while CAD software products provide only tolerance analysis or synthesis.

Monitoring the changes of individual forces and displacements, which have an impact on a major trigger force, may lead to the conclusion and the profile vs. time of their actions, or timing of all trigger mechanism parts. In this way, it is much easier to analyze the function of the arms and quickly select all illogical variations of parameters from analysis.

\section{ACKNOWLEDGMENT}

Results presented in this paper have been gathered within project No III 47029 financed by the Ministry of Science and Technology Development Republic of Serbia.

\section{REFERENCES}

[1] Sadílek, M., Čep, R., Budak, I., Soković, M. (2011). Aspects of using tool axis inclination angle. Strojniški vestnik - Journal of Mechanical Engineering, vol. 57, no. 9, p. 681-688, DOI:10.5545/sv-jme.2010.205.

[2] Pirnat, M., Savšek, Z., Boltežar, M., (2011). Measuring dynamic loads on a foldable city bicycle. Strojniški vestnik - Journal of Mechanical Engineering, vol. 57, no. 1, p. 21-26, DOI:10.5545/sv-jme.2009.149.

[3] Vitek, R., (2009). The generally unbalanced projectile load on the sporting rifle barrel. Proceedings of the $8^{\text {th }}$ WSEAS ICOSSSE, Genova.

[4] Vítek, R., Jedlička, L. (2010). Effect of the accuracy of target range measurement on the accuracy of shooting. Advances in Military Technology, vol. 5, no. 2, p. 6983.

[5] Macko, M. (1996). Synthesis of the trigger mechanisms according to the trigger characteristics. Proceedings of $1^{\text {th }}$ International Armament Conference, Military University of Technology, Solina, vol. 3, p. 55-60.

[6] Fišer, M., Lipták, P., Procházka, S., Macko, M., Jozefek, M. (2007.) Automatic Weapons. Alexander Dubček University of Trenčín, Trenčín.

[7] Macko, M. (2011). Influence of the size tolerances on the small arms trigger characteristic. Proceeding of OTEH, Belgrade. p. 228-232.

[8] Feng, C-X., Kusiak, A. (2000). Robust tolerance synthesis with the design of experiments approach. ASME Transactions: Journal of Manufacturing Science and Engineering, vol. 122, no. 3, p. 520-528, DOI:10.1115/1.1285860. 